NOTE

\title{
Pyrolysis Gas Chromatographic Characterization of Block Copolymers of Ordinary and Deuterated Styrenes
}

\author{
Hajime Ohtani, Shin Tsuge, ${ }^{*}$ Yuhshuh Matsushita, \\ and Mitsuru NAGASAWA \\ Department of Synthetic Chemistry, Faculty of Engineering, \\ Nagoya University, Nagoya 464, Japan.
}

(Received January 5, 1982)

\author{
KEY WORDS Pyrolysis Gas Chromatography / Glass Capillary Column / \\ Polystyrene / Deuterated Polystyrene / Poly(styrene(d)-b-styrene(h)) / \\ Poly(styrene(h)- $b$-styrene(d)- $b$-styrene(h)) /
}

It has been suggested that block copolymers of normal styrene $(\mathrm{St}(\mathrm{h}))$ and deuterated styrene (St(d)) play an important role in the conformation of polymer chains according to research utilizing the neutron scattering technique. ${ }^{1-3}$ The polymer samples for this purpose should have distributions as narrow as possible with respect to both molecular weight and composition. Thus, an accurate compositional analysis of the prepared block copolymers is important in subsequent quantitative conformational study. However, in both its physical and chemical properties, polystyrene(d) $(\operatorname{PSt}(\mathrm{d}))$ is quite comparable to polystyrene(h) (PSt(h)). Therefore, conventional elemental analysis has little application to these block copolymers. Also, NMR does not afford an accurate determination of the copolymer composition.

In this note, a new method is proposed for the determination of d-blocks in the di-block and triblock copolymers of $\mathrm{St}(\mathrm{h})$ and $\mathrm{St}(\mathrm{d})$ using pyrolysisgas chromatography (PGC). By this method, the dcontent in the block copolymers were determined with relative standard deviations of less than $3 \%$. Furthermore, the difference in thermal degradation behavior between PSt(d) and PSt(h) was examined.

\section{EXPERIMENTAL}

\section{Materials}

Two kinds of block copolymers, i.e., poly[St(d)-b-

Table I. Structure and average molecular weight characteristics of the samples

\begin{tabular}{clcc}
$\begin{array}{c}\text { Sample } \\
\text { No. }\end{array}$ & \multicolumn{1}{c}{ Structure } & $\begin{array}{c}\text { Number-average molecular } \\
\text { weight, } M_{n} \times 10^{-4}\end{array}$ & $\begin{array}{c}M_{w} / M_{n} \\
(\mathrm{GPC})^{\mathrm{b}}\end{array}$ \\
\hline & $\begin{array}{l}\text { (Precursor homopolymers) } \\
\text { PSt(d) }\end{array}$ & 3.3 & - \\
1 & PSt(h) & 14.0 & - \\
2 & PSt(h) & 3.0 & - \\
3 & (Block copolymers) & $31.2(3.3-27.9)^{\mathrm{a}}$ & - \\
4 & P(St(d)- $b$-St(h) & $30.2(14.0-3.0-13.2)^{\mathrm{a}}$ & 1.04 \\
5 & P(St(h)- $b$-St(d)- $b$-St(h)) & 41.6 & 1.03 \\
6 & Purified sample 4 & 29.3 & \\
7 & Purified sample 5 & & \\
\hline
\end{tabular}

a Values in the parentheses are the sequential $M_{n}$ for the corresponding blocks in the copolymers.

b Values of $M_{w} / M_{n}$ were obtained by gel-permeation chromatography (GPC).

* To whom correspondence should be addressed. 


\section{H. Ohtani et al.}

$\mathrm{St}(\mathrm{h})]$ and poly[St(h)- $b$-St(d)- $b$-St(h)], were synthesized by the sequential monomer addition technique in anionic polymerization with $s$-butyllithium in benzene. The details of the preparation method are described elsewhere. ${ }^{4}$ As comparative samples, the precursors were also taken at respective stages of the sequential polymerization. For comparison, purified samples of the block copolymers were prepared by precipitational fractionation using cyclo- hexane solution and changing temperature. The polymer samples so prepared are listed in Table I along with average molecular weight data. The number-average molecular weight $\left(M_{n}\right)$ of each sample was measured with a high-speed membrane osmometer. As a reference polymer for the calibration of PGC, a sample of poly- $\alpha$-methylstyrene (P $\alpha \mathrm{MSt}$ ) with $M_{n}=310,000$ was used.

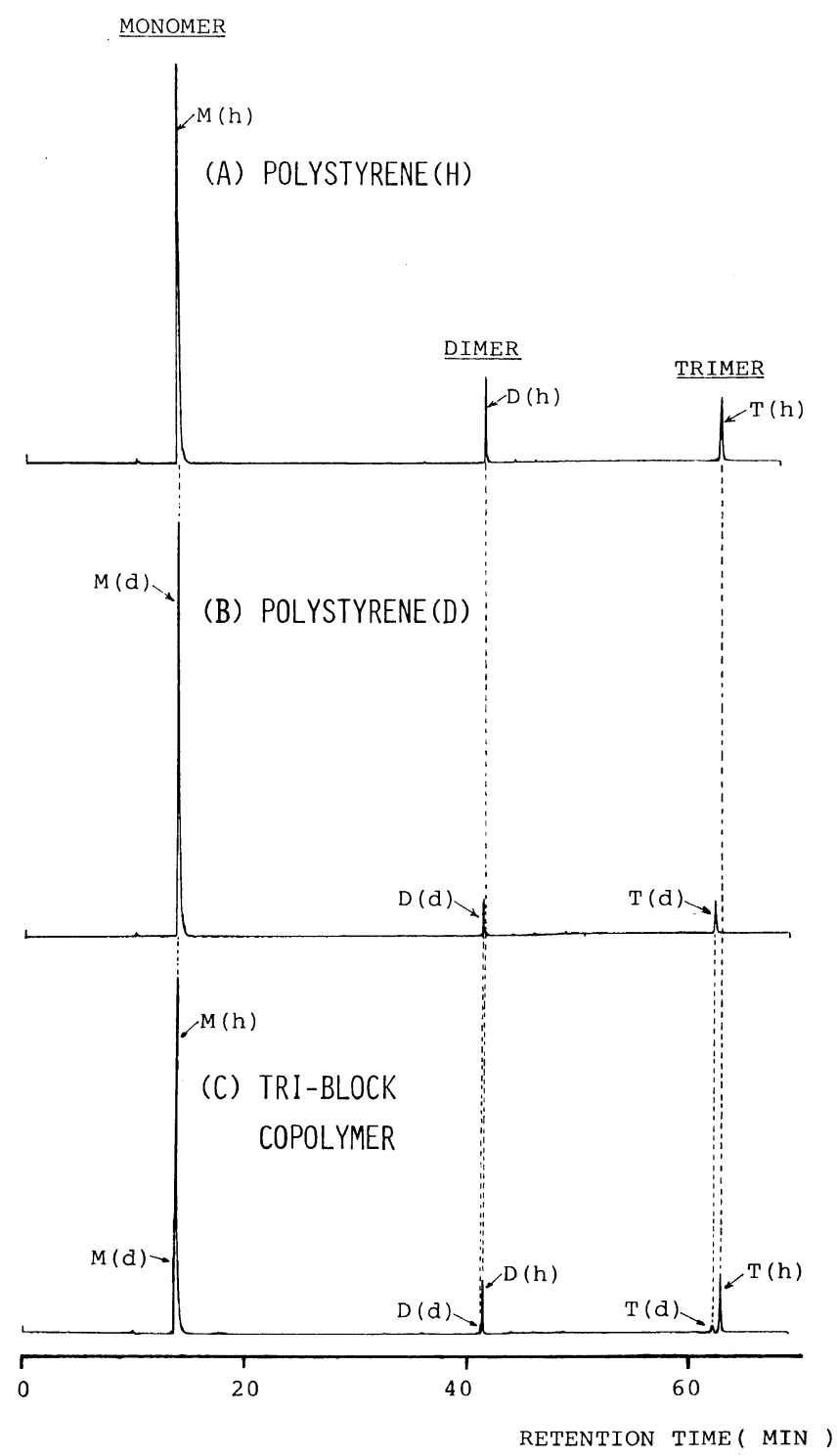

Figure 1. Pyrograms of two precursor homopolymers and a block copolymer at $510^{\circ} \mathrm{C}$ : (A) PSt(h) (sample 2), (B) PSt(d) (sample 1), (C) tri-block copolymer (sample 7 in Table I). M(h), D(h), T(h); M(d), $\mathrm{D}(\mathrm{d}), \mathrm{T}(\mathrm{d})$ : monomer, dimer, trimer of hydrogenated, and deuterated, respectively. 


\section{Conditions for PGC}

A Yanagimoto GP-1018 micro furnace-type pyrolyzer ${ }^{5}$ was directly attached to a Shimadzu $7 \mathrm{AG}$ gas chromatograph equipped with a flame ionization detector (FID). A glass capillary column (o.d. $0.9 \mathrm{~mm} \times$ i.d. $0.3 \mathrm{~mm} \times 40 \mathrm{~m}$ long) suspension coated with OV-101 and Silanox (325 mesh) was used in a temperature programming mode from 50 to $250^{\circ} \mathrm{C}$ at a rate of $10^{\circ} \mathrm{C} \mathrm{min}^{-1}$. The $50 \mathrm{ml} \mathrm{min}^{-1}$ nitrogen carrier gas flow at the pyrolyzer was reduced to $0.7 \mathrm{ml} \mathrm{min}^{-1}$ at the capillary column by a splitter $(70: 1)$. Samples ranging from 0.03 to $0.1 \mathrm{mg}$ were pyrolyzed at $510^{\circ} \mathrm{C}$ under the flow of the carrier gas. The peak areas of the resulting pyrograms were evaluated with a Yanagimoto System-1000 integrator.

\section{RESULTS AND DISCUSSION}

Figure 1 shows the pyrograms of the tri-block copolymer (sample 7) and those of the homopolymers as the associated precursors (samples 1 and 2). As was reported in our previous paper, ${ }^{6}$ the main degradation product from ordinary head-to-tail type PSts was the monomer. In addition, certain amounts of dimer and trimer also appeared on the pyrograms. Table II summarizes the retention data and the relative abundances of the degradation products from PSt(h)s and PSt(d).

\section{Retention Behavior of the Degradation Products}

It is of interest to note that the retention times of the deuterated products were always shorter than those of ordinary hydrogenated products. Since the stationary phase (OV-101) used for this separation eluted the samples almost in the order of their boiling points, this phenomenon suggests that, in spite of the increase in molecular weight by the increment of atomic weights in ${ }^{1} \mathrm{H}$ and ${ }^{2} \mathrm{D}$, the deuterated products always have lower boiling points than the corresponding hydrogenated products. The difference in boiling points may be due to the deuterium substitution of the relatively active hydrogen attached to the tertiary carbon in the polymer backbone.

The difference in the retention times among the monomers and dimers is so small that no complete separation of the associated peaks could be attained by a glass capillary column under the given chromatographic conditions. On the other hand, the trimer peaks were almost completely separated as shown in Figure 1(c).

\section{Relative and Absolute Abundances of the Degrada- tion Products}

$\mathrm{P} \alpha \mathrm{MSt}$, which is known to degrade into the monomer ( $\alpha$-methylstyrene) almost quantitatively at high temperature, ${ }^{7}$ can be used as a very good reference polymer for measuring the recovery of a given polymer sample with respect to the degradation products on the pyrogram. Table III summarizes the recovery data for $\operatorname{PSt}(\mathrm{h})$ and $\operatorname{PSt}(\mathrm{d})$ along with those for P $\alpha \mathrm{MSt}$. The molar sensitivity of a compound for FID is known to change as a simple function of the effective carbon number in the molecule. ${ }^{8}$ Therefore, the recovery ( $\%$ ) can be correlated to the total counts $\left(C_{\text {total }}\right)$ of the whole peaks up to $65 \mathrm{~min}$ on the pyrogram for a known amount $\left(W_{\mathrm{PSt}}\right)$ of PSt sample:

Table II. Retention data and relative abundance of the pyrolysis products from $\operatorname{PSt}(\mathrm{h})$ and $\operatorname{PSt}(\mathrm{d})$ at $510^{\circ} \mathrm{C}$

\begin{tabular}{|c|c|c|c|c|c|}
\hline \multirow{3}{*}{ Product } & \multirow{2}{*}{\multicolumn{2}{|c|}{$\begin{array}{l}\text { Retention } \\
\text { time/min }\end{array}$}} & \multicolumn{3}{|c|}{ Relative abundance $/ \mathrm{wt} \%$} \\
\hline & & & \multicolumn{2}{|c|}{ PSt(h) } & \multirow{2}{*}{$\begin{array}{c}\mathrm{PSt}(\mathrm{d}) \\
M_{n}=30,000\end{array}$} \\
\hline & $\mathrm{h}$ & $\mathrm{d}$ & $M_{n}=140,000$ & $M_{n}=30,000$ & \\
\hline Monomer & 13.8 & 13.7 & 78.1 & 77.2 & 87.9 \\
\hline Dimer & 41.6 & 41.4 & 7.2 & 6.8 & 3.9 \\
\hline Trimer & 63.1 & 62.4 & 11.2 & 10.6 & 6.3 \\
\hline The rest & - & - & 3.5 & 5.4 & 1.9 \\
\hline
\end{tabular}

\footnotetext{
${ }^{a} \mathrm{~h}$ and $\mathrm{d}$ represent the hydrogenated and deuterated forms, respectively.
} 
Table III. Recovery of the polymer samples as degradation products on the pyrogram

\begin{tabular}{|c|c|c|c|c|c|}
\hline \multirow{2}{*}{ Sample $^{\mathrm{a}}$} & \multirow{2}{*}{$\begin{array}{l}\text { Molecular } \\
\text { weight } \\
\text { of monomer }\end{array}$} & \multirow{2}{*}{$\begin{array}{c}\text { Effective }^{b} \\
\text { carbon } \\
\text { number }\end{array}$} & $\begin{array}{l}\text { Sample } \\
\text { size }\end{array}$ & \multirow{2}{*}{$\begin{array}{c}\text { Total peak }^{\mathrm{c}} \\
\text { intensity }\end{array}$} & \multirow{2}{*}{$\begin{array}{l}\text { Recovery of the sampled } \\
\text { on the pyrogram } \\
\%\end{array}$} \\
\hline & & & $\mu \mathrm{g}$ & & \\
\hline $\mathrm{P} \alpha \mathrm{MSt}$ & 120 & 9 & 189 & 358962 & $(100)$ \\
\hline $\operatorname{PSt}(\mathrm{h})$ & 106 & 8 & 183 & 359251 & 97.4 \\
\hline $\operatorname{PSt}(\mathrm{d})$ & 116 & 8 & 178 & 320264 & 97.1 \\
\hline
\end{tabular}

a $\operatorname{PSt}(\mathrm{h})=$ sample 2, and PSt $(\mathrm{d})=$ sample 1, in Table I.

b Effective carbon number for FID. ${ }^{8}$

c The integration of total peak intensity count up to the trimer region $(65 \mathrm{~min})$.

d Caluculated from eq 1 when the value for $\mathrm{P} \alpha \mathrm{MSt}$ is regarded as $100 \%$.

\section{Recovery $(\%)$}

$$
=\frac{W_{\mathrm{PSt}} /\left(\mathrm{MW} \text { of } \mathrm{St} \times C_{\mathrm{total}}\right)}{W_{\mathrm{P} \alpha \mathrm{MS}} /\left(\mathrm{MW} \text { of } \alpha \mathrm{MSt} \times C_{\alpha \mathrm{MSt}}\right)} \times \frac{8}{9} \times 100
$$

where $W_{\mathrm{P} \alpha \mathrm{MSt}}$ and $C_{\alpha \mathrm{MSt}}$ are the weight of $\mathrm{P} \alpha \mathrm{MSt}$ sample and the counts for the $\alpha$-methylstyrene peak on the pyrogram, respectively, and the factor $8 / 9$ is the ratio of the effective carbon numbers of styrene and $\alpha$-methylstyrene units for FID. The calculated recoveries, $97.4 \%$ for $\mathrm{PSt}(\mathrm{h})$ and $97.1 \%$ for $\operatorname{PSt}(\mathrm{d})$, are almost equal and may be regarded as highly quantitative under our pyrolysis-gas chromatographic conditions.

But, as shown in Table II, a fairly different tendency is observed for the relative abundance of the degradation products from PSt(h) and PSt(d). The most distinct feature is the monomer yield for PSt(d), which is significantly higher than that for $\mathrm{PSt}(\mathrm{h}) \mathrm{s}$. This is attributable to the higher depolymerization tendency for PSt(d) also caused by the ${ }^{2} \mathrm{D}$ substitution of the active hydrogen attached to the tertiary carbon. As was described in our previous work, ${ }^{9}$ only slight differences could be observed for the data of PSt(h) with $M_{n}=30,000$ and PSt(h) with $M_{n}=140,000$, this was due to the fact that the molecular weight effects on the thermal degradation of PSt became almost invariable above $M_{n}=30,000$.

\section{Determination of Deuterated Block Content in the Copolymers}

The recovery data shown in Table III suggest that the block content in the block copolymers may be discussed quantitatively provided the total count of the associated peaks up to the trimer region is
Table IV. The content of d-blocks in the copolymers

\begin{tabular}{ccc}
\hline & \multicolumn{3}{c}{ Contents of d-block $/ \mathrm{mol}^{\circ} \%$} \\
\cline { 2 - 3 } Sample No. & Obsd by PGC & Calcd $^{\mathrm{a}}$ \\
\hline 4 & 11.2 & $10.6(10.3)$ \\
5 & 10.7 & $9.9(10.1)$ \\
7 & 6.3 & - \\
\hline
\end{tabular}

a Values were calculated by the $M_{n}$ data in Table I, and those in the parentheses were estimated from the content of monomer and initiator for polymerization.

considered. Although the pyrograms of the copolymers were resolvable only for the trimer peaks, the total count of the associated peaks from the relative peak intensity of d-trimer and h-trimer, using the relative abundance data of the monomers, dimers and trimers from the precursor homopolymers (Table II). Thus, the d-block content in the copolymer may be expressed as follows:

d-block content $(\mathrm{mol} \%)$

$$
=\frac{Y_{\text {d-trimer }} / 0.063}{Y_{\text {d-trimer }} / 0.063+Y_{\text {h-trimer }} / 0.112} \times 100
$$

where $Y_{\mathrm{d}-t \text { rimer }}$ and $Y_{\mathrm{h}-\text { trimer }}$ are the relative yields of $\mathrm{d}$-trimer and h-trimer $\left(Y_{\mathrm{d} \text {-trimer }}+Y_{\mathrm{h} \text {-trimer }}=1\right)$, and 0.063 and 0.112 are the relative abundance of the $d-$ trimer and h-trimer from PSt(d) and PSt(h), respectively (Table II).

The observed results so obtained are given in 
Table IV. In this method, the relatively small trimer yields had to be used for the determination of the d-block content. However, the relative standard deviation was within $3 \%$. If a more efficient column is used to bring about a complete separation of the main peaks (monomers), results of greater reproducibility would be obtained.

The d-block content both in the di-block copolymer (sample 4) and the tri-block copolymer (sample 5) prior to fractional purification are in fairly good agreement with the values calculated from the copolymerization data. However, following purification, the d-block content in the diblock copolymer drastically changes to the lower value of $6.3 \mathrm{~mol} \%$. From the ultracentrifugal schlieren patterns of the di-block copolymers before and after fractionation, it was found that the lower molecular weight fractions removed by fractionation should consist primarily of the deuterated homopolymer. ${ }^{4}$

\section{REFERENCES}

1. J. P. Cotton, D. Decker, H. Benoit, B. Farnoux, J. Higgins, G. Jannink, R. Ober, C. Picot, and J. des Cloizeaux, Macromolecules, 7, 863 (1974).

2. M. Daoud, J. P. Cotton, B. Farnoux, G. Jannink, G. Sarma, H. Benoit, R. Duplessix, C. Picot, and P. G. de Dennes, Macromolecules, 8, 804 (1975).

3. R. Duplessix, J. P. Cotton, H. Benoit, and C. Picot, Polymer, 20, 1181 (1979).

4. Y. Matsushita, H. Furuhashi, H. Choshi, I. Noda, M. Nagasawa, T. Fujimoto, and C. C. Han, Polym. $J .$, 14, 489 (1982).

5. S. Tsuge and T. Takeuchi, Anal. Chem., 49, 348 (1977).

6. Y. Sugimura, T. Nagaya, and S. Tsuge, Macromolecules, 14, 520 (1980).

7. T. Okumoto and T. Takeuchi, Bull. Chem. Soc. Jpn., 46, 1717 (1973).

8. J. C. Sternberg, W. S. Gallaway', and D. T. L. Jones, "Gas Chromatography," ed. by N. Brenner, et al., Academic Press, New York, N.Y., 1962.

9. S. Tsuge, T. Okumoto, and T. Takeuchi, $J$. Chromatogr. Sci., 7, 250 (1969). 